\title{
The Analysis of Multichannel Dual Clock Probability Detection CSMA Random Multiple Access
}

\author{
Zhi Xul,a ${ }^{1, \text { Hongwei Ding }}{ }^{1, b}$, Shengjie Zhou ${ }^{1}$, Longjun Liu ${ }^{1}$ \\ ${ }^{1}$ School of Information, Yunnan University, Kunming, Yunnan 650504, China \\ a775862901@qq.com, bdhw1964@163.com
}

\begin{abstract}
Keywords: carrier sense multiple access; Quality of Service; discrete continuous clock strategy; priority; throughput
\end{abstract}

\begin{abstract}
Carrier Sense Multiple Access (CSMA) is a very important network communication protocol. According to the different ways of service agreement can be divided into: No-Persistent CSMA, 1-Persistent CSMA, P- Persistent CSMA. Although these protocols can improve the system throughput, but there are also some shortcomings, they are not fully utilized in the channel idle period, cannot provide the corresponding needs for various services. Therefore, from the point of view of improving the Quality of Service (QoS) of wireless communication network and utilization of channel resource. the Multichannel Dual Clock Probability Detection CSMA Random Multiple Access was proposed. In the protocol, the discrete continuous clock strategy was used to improve the QoS of each service in the wireless communication network. Based on the mathematical model, the throughput of the system and priority is obtained; Finally, the simulation shows that the new protocol can make use of the idle time of the channel, and achieve the system load balance by controlling the arrival rate of the priority, which ensures the QoS requirements of the high priority service.
\end{abstract}

\section{Introduction}

With the rapid development of wireless communication, the types of users are increasing, and the user's individual requirements are becoming more and more diversified. The first communications industry is just a few voice services, and now developed to the data, voice and video and other services coexist. In order to meet the quality of service, many new technologies have been put into practical application, only rely on the traditional communication protocol has been unable to meet the different needs of all users.

Compared with the traditional CSMA ${ }^{[1,2,3]}$, the hybrid control dual clock random access protocol has many advantages such as high flexibility, higher channel utilization, shorter channel access delay. Therefore, in order to make better use of wireless cyber source, based on the wireless communication network as the research object, put forward the Multichannel Dual Clock Probability Detection CSMA Random Multiple Access, which combines the advantages of discrete continuous hybrid $^{[4]}$ system, through mathematical analysis model, the theoretical formula was then compared with the new agreement; A variety of other protocol performance is obtained by simulation analysis. The results show that the proposed new protocol throughput performance, load capacity and channel access efficiency of the new protocol is better than the other, is a respected and excellent communication network protocol.

\section{Model introduction}

\subsection{Multichannel model}

This paper studies the multichannel protocol, in the wireless communication system, there are $\mathrm{N}$ channels for all end users to occupy randomly, at the same time, the terminal uses the Multichannel Dual Clock Probability Detection CSMA Random Multiple Access technology. 


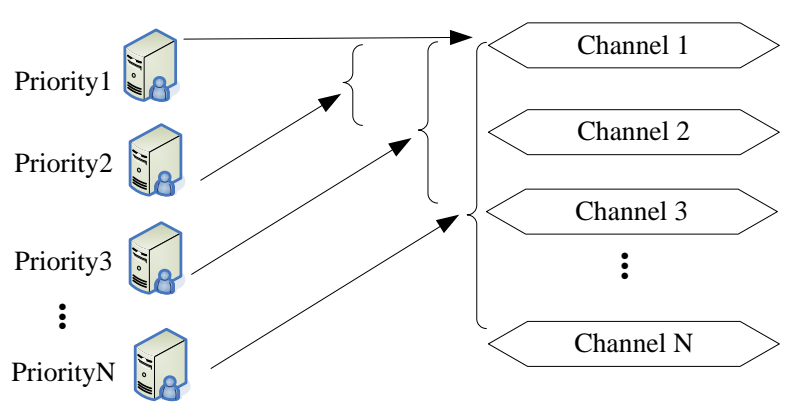

Fig.1. load balancing system model

Figure 1 is the load balancing system model, the number of channels in the system is $\mathrm{N}$, and there are $\mathrm{N}$ priority. Priority order from low to high order priority 1, priority level 2...priority $\mathrm{N}$, and the number of users is unlimited in each priority ${ }^{[5]}$. If a user is in priority $\mathrm{x}$, then the service is fixed to occupy channel 1 to channel $\mathrm{X}$. The priority of the $\mathrm{Y}$ on channel $\mathrm{x}$ arrival rate is $\lambda_{y}=$ $\frac{G_{x}}{N-x+1}$, the load balancing system, each channel utilization rate was $G_{x}=G(x=1,2, \ldots, N)$.

\subsection{The throughput analysis of new protocol}

Before the analysis of system performance, there are some assumptions:

(1) The access mode of the system is the new protocol, and the arrival process of the information packet on the channel $\mathrm{x}$ is the Poisson process with independent parameter of $G_{x}$;

(2) When the channel is idle (I event), the length of the slot is a, and the length of the slot is 1 when the message is sent (BU event);

the number of users in per priority is unlimited, priority $\mathrm{y}$ on channel $\mathrm{x}$ arrival rate is $\lambda_{x}=\frac{G_{x}}{N-x+1}$

(3) The channel is assumed to be ideal and there is no noise interference in the channel.

(4)The probability of no packet arrival in the channel is $e^{-P(1+a) G}$. So the average slot length of each state in the channel can be derived.

First we want to calculate the average length of the message packet in the channel $\mathrm{x}$ to be successfully transmitted $\mathrm{E}\left[U_{x}\right]$; The system in the $\mathrm{X}$ channel, the information packet which you want to send success, there will be two cases:

The last time slot in the idle period has only one message arrive, then the next time slot will sent this message successfully, the event is denoted as $U_{x 1}$, the average length of $U_{x 1}$ is:

$\mathrm{E}\left(U_{x 1}\right)=\frac{a G_{x} e^{-a G_{x}}}{1-\mathrm{e}^{-a G_{x}}}$

Information arrive in a busy period (TP), and this information is the only one in the current TP which listen to the information packet by the probability $\mathrm{P}$, and the information will be sent in the next time slot, this event is recorded as $U_{2}$.

$\mathrm{E}\left(U_{2}\right)=\sum_{i=1}^{\infty} \sum_{j=1}^{\infty} \sum_{k=0}^{i-1} k P(i, j)=P(1+a) G_{x}$

$\mathrm{E}\left(U_{x}\right)=\mathrm{E}\left(U_{x 1}\right)+\mathrm{E}\left(U_{x 2}\right)=\frac{a G_{x} e^{-a G_{x}}}{1-\mathrm{e}^{-a G_{x}}}+P(1+a) G_{x}$

Secondly, the length of the busy period $\mathrm{BU}$ in the channel $\mathrm{x}$ is analyzed:

$\mathrm{E}\left(\mathrm{B} U_{x}\right)=\sum_{i=1}^{\infty} \sum_{j=1}^{\infty}(1+a) * i * P(i, j)=\frac{1+a}{e^{-P(1+a) G_{x}}}$

Finally, the analysis of the average length of the idle period $I_{x}$ in the $\mathrm{x}$ channel $\mathrm{E}\left(I_{x}\right)$; In the idle period, the I time slot satisfies the geometric distribution of $\mathrm{E}(\mathrm{N})=\frac{1}{1-a G_{x}}$. Then there is:

$\mathrm{E}\left(I_{x}\right)=\mathrm{a}\left(\frac{1}{1-e^{-a G_{x}}}-1\right) \mathrm{a}+\frac{a G_{x} e^{-a G_{x}}}{1-e^{-a G_{x}}} * \frac{a}{2}+\frac{1-a G_{x} e^{-a G_{x}}-e^{-a G_{x}}}{1-e^{-a G_{x}}} * \frac{a}{2}$

All channels in the system, the arrival rate are equal because the channel load balance. So the $G_{1}=G_{2}=. .=G_{N}=\mathrm{G}$. So we can sum up the throughput on all channels, the $\mathrm{S}$ is:

$\mathrm{S}=\sum_{x=1}^{N} \frac{E\left(U_{x}\right)}{E\left(I_{x}\right)+E\left(B U_{x}\right)}$ 
If the average length of the message packet which is assumed to transmit the priority $\mathrm{y}$ in the period of the channel $\mathrm{x}$ is $\mathrm{E}\left(U_{x}^{p_{y}}\right)$. Since the channel load is balanced, the arrival rate of priority $\mathrm{Y}$ in the channel $\mathrm{x}$ is: $\lambda_{x}^{p_{y}}=G_{x} /(N-x+1)$.Then the same way:

$\mathrm{E}\left(U_{x}^{\left(p_{y}\right)}\right)=\frac{\lambda_{x}^{p_{y}}}{G_{x}} E\left(U_{j}\right)=\frac{1}{N-x+1}\left(\frac{a G_{x} e^{-a G_{x}}}{1-\mathrm{e}^{-a G_{x}}}+P(1+a) G_{x}\right)$

According to the arrival rate of $\mathrm{G}$, the throughput of priority $\mathrm{Y}$ can be obtained:

$S_{P y}=\left(\sum_{x=1}^{y} \frac{1}{N-x+1}\right) \frac{G e^{-(1+a) G}\left(1+a-e^{-a G}\right)}{(1+a)\left(1-e^{-a G}\right)+a e^{-(1+a) G}\left(1-\frac{1}{2} a G e^{-a G}\right)}$

\section{Simulation and results analysis}

Based on the above analysis, the new communication system is simulated by Matlab simulation software. The simulation results are as follows.

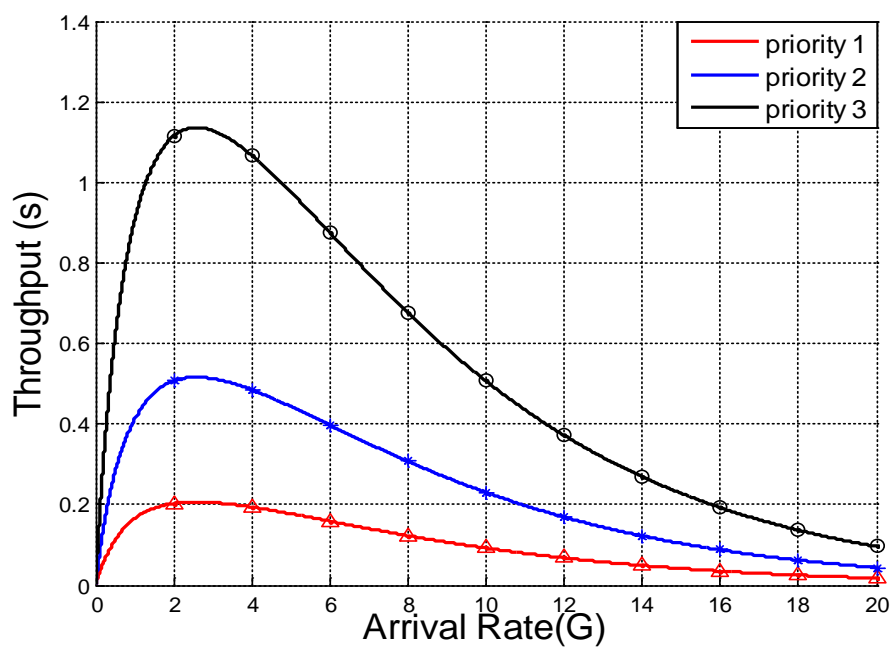

Figure 2: Situation of priority S-G when channel number is 3
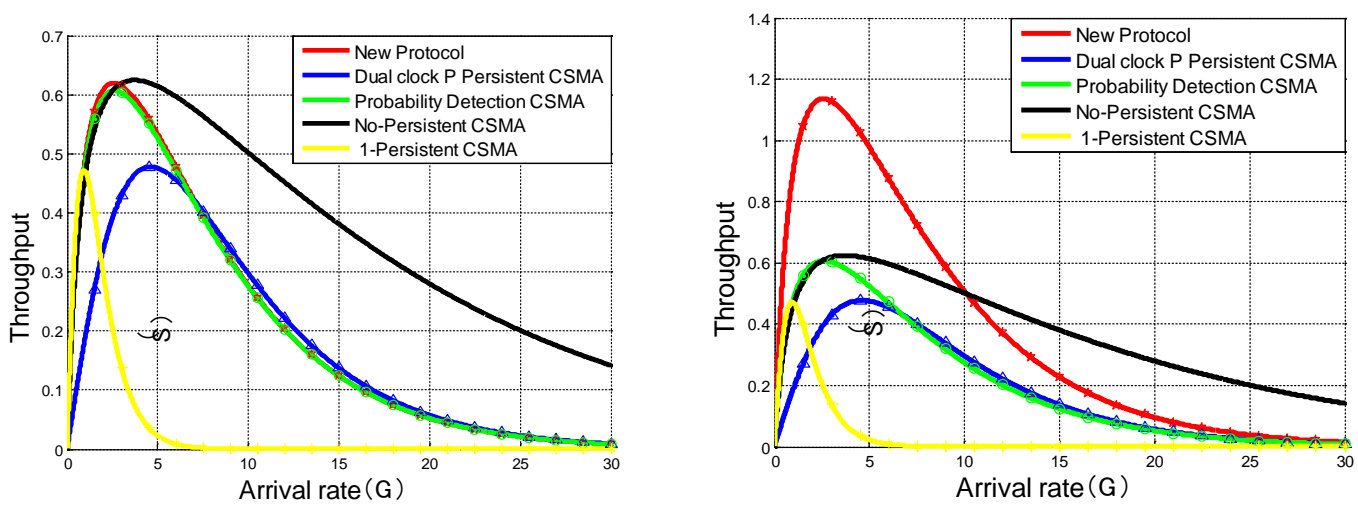

Figure 3: Comparison of throughput of each CSMA protocol

(Single channel and Three channel)

From the simulation results, the following conclusions can be drawn:

1. As we can be seen from Figure2, when the number of channels is 3, the priority traffic throughput will decrease gradually with the increase of the load, and the high priority can always get higher throughput.

2. From Figure3, the new protocol combines with the advantages of continuous system and discrete system and reduce the idle rate in single channel, so it will be assigned to more information packets to send, so the throughput of new protocol is slightly higher than Probability Detection CSMA. With the increase of the number of channels, the system can allocate channel resources according to different services, so when the number of channels is 3 , 
the throughput of the new protocol has been significantly higher than other protocols.

\section{Conclusion}

In this paper, the Multichannel Dual Clock Probability Detection CSMA Random Multiple Access was proposed, which based on the single strategy $\mathrm{P}$ detection CSMA protocol. It is a combination of time slot and continuous analysis methods, and divides the number of QoS in the network by priority. In this paper, the mathematical modeling and simulation by MATLAB. Finally, the simulation is drawn that the protocol has excellent communication performance, which can be used in the wireless communication network.

\section{Acknowledgments}

Supported by the National Natural Science Foundation of China
$(61461053,61461054,61072079) ;$

\section{References}

[1] Yun Tang, Dongfeng Zhao, Hongwei Ding. Multi-channel 1 adhere to the protocol analysis of -CSMA multi access communication system [J]. computer engineering and application, 2005, 35:106-108.

[2] Lili Zheng, Dongfeng Zhao, Hongwe Ding. Performance analysis of multi-channel 1-CSMA protocol [J]. Journal of Yunnan University, 2006,28 (4): 307-311.

[3] Shengjie Zhou et al., Research on the Discrete time Three-Dimensional Probability Csma Protocol In ad-hoc Network. International Journal of Recent Scientific Research Vol. 6, Issue, 5, pp.4257-4262, June, 2015.

[4] Tan Mingchuan. Analysis of CSMA/CA wireless sensor network protocol and control circuit based on probability detection [D]. Yunnan University, 2011.

[5] Guo Yingying. Analysis and research of BTMC-CSMA protocol with in wireless Ad Hoc network [D]. Yunnan University.2014. 